\title{
Copper-zinc superoxide dismutase (Sod1) activation terminates interaction between its copper chaperone (Ccs) and the cytosolic metal-binding domain of the copper importer Ctr1
}

\author{
Amélie Skopp • Stefanie D. Boyd • Morgan S. Ullrich • Li Liu • \\ Duane D. Winkler $(\mathbb{D})$
}

Received: 7 May 2019/Accepted: 3 July 2019/Published online: 10 July 2019

(C) The Author(s) 2019

\begin{abstract}
Copper-zinc superoxide dismutase (Sod1) is a critical antioxidant enzyme that rids the cell of reactive oxygen through the redox cycling of a catalytic copper ion provided by its copper chaperone (Ccs). Ccs must first acquire this copper ion, directly or indirectly, from the influx copper transporter, Ctr1. The three proteins of this transport pathway ensure careful trafficking of copper ions from cell entry to target delivery, but the intricacies remain undefined. Biochemical examination of each step in the pathway determined that the activation of the target (Sod1) regulates the $\mathrm{Ccs} \cdot \mathrm{Ctr} 1$ interaction. Ccs stably interacts with the cytosolic C-terminal tail of $\mathrm{Ctr} 1(\mathrm{Ctr} 1 \mathrm{c})$ in a copper-dependent manner. This interaction becomes
\end{abstract}

Amélie Skopp and Stefanie D. Boyd have contributed equally to this manuscript

Protein Accession IDs Human Ccs (UniProtKB-O14618), Human Sod1 (UniProtKB-P00441), Human Ctr1 (UniProtKB-O15431)

Electronic supplementary material The online version of this article (https://doi.org/10.1007/s10534-019-00206-3) contains supplementary material, which is available to authorized users.

A. Skopp · S. D. Boyd · M. S. Ullrich · L. Liu

D. D. Winkler $(\square)$

Department of Biological Sciences, The University of

Texas at Dallas, 800 W. Campbell Road, Richardson,

TX 75080, USA

e-mail: duane.winkler@utdallas.edu tripartite upon the addition of an engineered immature form of Sod1 creating a stable $\mathrm{Cu}(\mathrm{I})-\mathrm{Ctr} 1 \mathrm{c} \cdot \mathrm{Ccs} \cdot \mathrm{Sod} 1$ heterotrimer in solution. This heterotrimer can also be made by the addition of a preformed Sod1.Ccs heterodimer to $\mathrm{Cu}(\mathrm{I})-\mathrm{Ctr} 1 \mathrm{c}$, suggestive of multiple routes to the same destination. Only complete Sod1 activation (i.e. active site copper delivery and intrasubunit disulfide bond formation) breaks the Sod1.Ccs.Ctr1c complex. The results provide a new and extended view of the Sod1 activation pathway(s) originating at cellular copper import.

Keywords Ctr1 1 Sod $1 \cdot$ Ccs $\cdot$ Copper-trafficking · Copper chaperone

\section{Introduction}

Copper is a critical cofactor for many enzymes that take advantage of its redox activity to catalyze a wide range of chemical reactions. Proper levels of copper are required throughout the cell for processes such as respiration, enzymatic catalysis, and neurotransmitter synthesis (Bremner 1998). Unregulated copper-based reactions are detrimental to the cell by generating free radical oxygen species (Halliwell and Gutteridge 1990; Pena et al. 1999). Therefore, aerobic organisms have evolved a tightly controlled network of copper trafficking molecules for import, shuttling, and 
delivery to specific targets across the cellular landscape (reviewed in Rosenzweig and O'Halloran 2000; Rosenzweig 2001; Robinson and Winge 2010).

Copper ion import is facilitated by the Ctr family of integral membrane transporters that bring copper into the cytoplasm in an ATP-independent fashion (Dancis and Haile 1994; Lee et al. 2002; Kim et al. 2008). Members of the Ctr-family, of which Ctr1 is the most prominent, are small transmembrane proteins consisting of a conserved extracellular N-terminal domain (ecto/NTD), three transmembrane domains (TMDs), and a short C-terminal tail ( $\mathrm{Ctr} 1 \mathrm{c})$, which is the only domain that fully extends into the cytosol (Eisses and Kaplan 2002; Puig et al. 2002; Klomp et al. 2003). Trimerization of Ctr 1 monomers forms a pore through which $\mathrm{Cu}(\mathrm{I})$ (i.e. reduced/cuprous copper) is transported into the cell (De Feo et al. 2009). $\mathrm{Cu}(\mathrm{I})$ is then simultaneously coordinated by three $\mathrm{Ctr} 1 \mathrm{c}$ tails, each containing an $\mathrm{HCH}$ copper binding motif. Binding of $\mathrm{Cu}(\mathrm{I})$ to a single $\mathrm{HCH}$ at one tail is possible, however normal $\mathrm{Cu}(\mathrm{I})$-binding motifs involve two or three cysteine residues coordinating $\mathrm{Cu}(\mathrm{I})$. (Pickering et al. 1993) By holding the $\mathrm{Cu}(\mathrm{I})$ between multiple tails, $\mathrm{Ctr} 1$ can mimic the more favorable $\mathrm{Cu}(\mathrm{I})$ coordinating motifs. The $\mathrm{Cu}(\mathrm{I})$ is then offered to cytosolic copper traffickers (Kahra et al. 2016). Two ATP-dependent pumps ATP7A and ATP7B that reside in the membranes of the trans-Golgi network, handle copper ion export (Lutsenko et al. 2002; Voskoboinik and Camakaris 2002).

A diverse family of intracellular copper binding proteins termed "copper chaperones" transport cytosolic copper ions to dedicated protein targets (O'Halloran and Culotta 2000; Kahra et al. 2016; Kaplan and Maryon 2016). One such molecule is the copper chaperone for Sod1 (Ccs) that has been shown to be critical for efficient activation of the ubiquitous anti-oxidant enzyme copper-zinc superoxide dismutase (Sod1) (Culotta et al. 1997; Schmidt et al. 2000; Wong et al. 2000; Fetherolf et al. 2017a, b). Ccs proteins consist of three conserved domains that are essential for target recognition and copper ion delivery (reviewed in Fetherolf et al. 2017a, b). Unlike other copper chaperones, Ccs also plays a crucial role in the formation of an intra-subunit disulfide bond within Sod1 (reviewed in Fetherolf et al. 2017a, b). The $\mathrm{N}$-terminal domain (D1) contains a MxCxxC copperbinding motif and is structurally similar to another copper chaperone that guides copper to the aforementioned secretory pathway (Atx1/Atox1) (Xiao and Wedd 2002; Hussain et al. 2008; Rodriguez-Granillo and Wittung-Stafshede 2008; Hussain et al. 2009; Rodriguez-Granillo and Wittung-Stafshede 2009; Rodriguez-Granillo et al. 2010). The second domain (D2) is analogous to Sod1 in both sequence and structure (Lamb et al. 1999) and plays a key role in Sod1 binding (Lamb et al. 2000, 2001; Winkler et al. 2009; Fetherolf et al. 2017a, b). The C-terminal domain (D3) possesses an invariant $\mathrm{CxC}$ motif known to bind copper and is essential for complete Sod1 activation in vivo (Schmidt et al. 1999). Though multiple three-dimensional structures of Ccs (Lamb et al. 1999, 2000; Banci et al. 2013) and complete Ccs.Sod 1 complexes have been determined (Lamb et al. 2001; Fetherolf et al. 2017a, b; Sala et al. 2019) a complete molecular mechanism for Ccsmediated Sod1 activation is tenuous and remains under debate.

Even less understood is how, where, or when copper chaperones, including Ccs, acquire their copper cargo from the cell (Flores and Unger 2013). Indeed, the passage of $\mathrm{Cu}(\mathrm{I})$ from the cytosolic $\mathrm{Ctr} 1 \mathrm{c}$ domain to Ccs still requires clarity, while related work implies that the reduced form of glutathione (GSH) may play an intermediary role (Maryon et al. 2013; Pope et al. 2013). Although Ctr1 has been studied extensively for years, an initial model for Ctr1mediated $\mathrm{Cu}(\mathrm{I})$ import is only beginning to emerge (reviewed in Ohrvik and Thiele 2014).

Here, we demonstrate a direct $\mathrm{Cu}(\mathrm{I})$-dependent interaction between human Ctr1c and Ccs. Unexpectedly, immediate copper transfer and dissolution of the $\mathrm{Cu}(\mathrm{I})-\mathrm{Ctr} 1 \mathrm{c} \cdot \mathrm{Ccs}$ complex did not occur. Furthermore, a stable $\mathrm{Cu}(\mathrm{I})-\mathrm{Ctr} 1 \mathrm{c} \cdot \mathrm{Ccs} \cdot \mathrm{Sod} 1$ heterotrimeric complex is observed when copper delivery and/or disulfide bond formation in Sod1 (i.e. Sod1 activation) is stalled. $\mathrm{Cu}(\mathrm{I})-\mathrm{Ctr} 1 \mathrm{c}$ will readily bind either $\mathrm{Ccs}$ or a pre-formed Ccs-Sod1 complex, which backs related data showing that the copper binding status of Ccs does not affect its affinity for immature Sod1 (Luchinat 2017; Boyd et al. 2018). Complete Sod1 activation (i.e. zinc and copper binding along with disulfide oxidation $\left(\mathrm{Cu}, \mathrm{Zn}\right.$-Sod1 $\left.{ }^{\mathrm{SS}}\right)$ ) dissociates the heterotrimeric complex into a mixture of its own parts (Banci et al. 2012; Wright et al. 2016). The data presented here builds a comprehensive molecular mechanism for the direct Ctr1-to-Ccs-to-Sod1 copper influx pathway 
and suggests that the entire pathway can proceed within a single macromolecular complex.

\section{Materials and methods}

\section{Materials}

The C-terminal 13 amino acids of the human Ctr1 $\mathrm{Cu}$ (I)-transporter (Ctr1c), KKAVVVDITEHCH, were custom-ordered as a lyophilized peptide from Sigma. DTT (dithiotreitol), TCEP-HCl (tris(2-carboxyethyl)phosphine), and IPTG (isopropyl 1-thio- $\beta$ D-galactopyranoside) were purchased from GoldBio. Tris-base, mono- and dibasic sodium phosphate, sodium acetate, ammonium persulfate (APS), EDTA, $\beta$-mercaptoethanol (BME), and sodium chloride were acquired from Fisher. Nitroblue terazolium and bathocuproinedisulfonic acid (BCS) were purchased from Alfa Aesar. Primers for site-directed mutagenesis and zinc sulfate heptahydrate were bought from Sigma. Maleimide-polyethyleneglycol 2000 was purchased from nanocs. Tetramethylethylenediamine (TEMED) was purchased from Thermo Scientific, while riboflavin was acquired from Acros Organics. Alexa-488-succinimidyl ester was purchased from Life Technologies. $\mathrm{Cu}(\mathrm{I})-\left(\mathrm{CH}_{3} \mathrm{CN}_{4}\right) \mathrm{PF}_{6}$ was purchased from Strem Chemicals. His-Trap Nickel affinity columns, SQ (anion exchange) columns, and gel filtration columns were purchased from GE LifeSciences.

Ccs1 and Sod1 cloning, mutagenesis, expression, and purification

Human wild-type (WT) Ccs1 (UniProtKB-O14618) was cloned into a pAG8H vector containing an inducible lacZ site, $\mathrm{N}$-terminal $\mathrm{His}_{8}$-tag, and an internal tobacco etch virus (TEV) cleavage site using NarI and SalI restriction sites. Sod1 WT (UniProtKB_-P00441) was cloned using NarI and HindIII sites into the same vector. Mutations in Ccs and Sod1 were generated using the Thermo Scientific sitedirected mutagenesis kit according to the provided protocol. Escherichia coli BL21 DE3 PLysS cells (purchased from Promega) were transformed and grown at $37{ }^{\circ} \mathrm{C}$ in $2 \mathrm{xYT}$ medium to an $\mathrm{A}_{600 \mathrm{~nm}}$ of 0.6-0.9 and induced with 3-5 mM IPTG. After an additional $4 \mathrm{~h}$ of growth cells were harvested and purified using a His-Trap $\mathrm{HP} \mathrm{Ni}^{2+}$ affinity column by GE Healthcare. The His $_{8}$-tag was removed from the purified protein by digestion at room temperature overnight with TEV protease engineered to contain a non-cleavable $\mathrm{His}_{8}$-tag. The resulting cleaved $\mathrm{His}_{8}$-tag as well as the TEV protease were removed from Ccs1 by another $\mathrm{Ni}^{2+}$ affinity purification.

Protein metallation

Sod1 was stripped of metals by incubation in Sod1 stripping buffer 1 (50 mM NaOAc pH 3.5, $10 \mathrm{mM}$ EDTA, $10 \mathrm{mM}$ DTT) for $4 \mathrm{~h}$ at room temperature and then transferred to metal-free buffer $2(50 \mathrm{mM} \mathrm{NaOAc}$ $\mathrm{pH} 5.5,10 \mathrm{mM}$ DTT) overnight at $4{ }^{\circ} \mathrm{C}$. The metal free protein was buffer exchanged into $50 \mathrm{mM}$ Tris $\mathrm{pH}$ 8. Ccs1 was stripped of metals by incubation in Ccs1 stripping buffer (20 mM Tris $\mathrm{pH} 8,10 \mathrm{mM}$ EDTA, $10 \mathrm{mM}$ DTT) overnight at $4{ }^{\circ} \mathrm{C}$. The metal-free protein was buffer exchanged into either metal free $50 \mathrm{mM}$ Tris $\mathrm{pH}$. All proteins were $\mathrm{Cu}(\mathrm{I})$ loaded anaerobically using $\mathrm{Cu}-\mathrm{PF}_{6}$. Metal loading was confirmed by induced coupled plasma mass spectrometry (ICP-MS) using the Agilent 7900 facility here at UTD. Samples for ICP-MS were digested with $1 \% \mathrm{HNO}_{3}$ for analysis and performed in triplicate. The buffer in these experiments was measured for baseline metal content. Sod1 activity assays were performed as described previously (Fetherolf et al. 2017a, b).

$\mathrm{Cu}(\mathrm{I})$-loaded $\mathrm{Ctr} 1 \mathrm{c}$ production

Apo-Ctr1c was quantified by amino acid analysis by AAA Service Laboratory, Inc. Absorbance was measured from 200 to $360 \mathrm{~nm}$ using a Cary300 UV-Vis spectrophotometer by Agilent and the extinction coefficient was calculated. The Ctr1c-peptide was $\mathrm{Cu}(\mathrm{I})$-loaded anaerobically in equimolar ratio with $\mathrm{Cu}(\mathrm{I})-\left(\mathrm{CH}_{3} \mathrm{CN}_{4}\right) \mathrm{PF}_{6}$ for $2 \mathrm{~h}$ and excess unbound $\mathrm{Cu}(\mathrm{I})$ was removed by size-exclusion chromatography using a BioRad P2 column in spectroscopy buffer or by dialysis. ICP-MS results indicated that one $\mathrm{Cu}(\mathrm{I})$ ion was coordinated by $3 \mathrm{Ctr} 1 \mathrm{c}$ peptides, similar to what is seen in full-length Ctr1 (De Feo et al. 2009). Even when copper was added in 3-5 fold excess of peptide, the resulting copper bound remained consistent. 
Amine-specific labeling of Ctr1c

Apo and $\mathrm{Cu}(\mathrm{I})-\mathrm{Ctr} 1 \mathrm{c}$ were incubated with equimolar amounts of Alexa-488 succinimidyl ester by Fisher at room temperature in $\mathrm{pH} 7.4$ buffer for $1 \mathrm{~h}$. The labeling reaction was quenched with $1 \mathrm{M}$ Tris $\mathrm{pH} 8$ was then dialyzed overnight at $4{ }^{\circ} \mathrm{C}$ to remove excess dye and its metal status was confirmed by assaying its ability to provide $\mathrm{Cu}(\mathrm{I})$ to $\mathrm{Sod} 1$.

Nickel pulldown assay

His 8 -tagged WT and mutant Ccs1 and Sod1 variants were expressed and purified by Ni-affinity chromatography. 5uM bait protein was incubated with $40 \mu \mathrm{L} \mathrm{Ni-}$ NTA agarose bead slurry in $20 \mathrm{mM}$ Tris pH 7.5, $150 \mathrm{mM} \mathrm{NaCl}, 2 \mathrm{mM}$ TCEP-HCl, and $200 \mu \mathrm{M}$ BCS for $1 \mathrm{~h}$ at room temperature. $15 \mathrm{uM} \mathrm{Cu}(\mathrm{I})-\mathrm{Ctr} 1 \mathrm{c}_{488}$ was incubated with the bait proteins at room temperature for $1 \mathrm{~h}$ and washed three times with $500 \mathrm{uL} 20 \mathrm{mM}$ Tris $\mathrm{pH} 7.5,150 \mathrm{mM} \mathrm{NaCl}, 2 \mathrm{mM}$ TCEP-HCl, and $100 \mathrm{mM}$ imidazole. Proteins for SDS-PAGE analysis were eluted by the addition of $2 \mathrm{X}$ Laemmli dye with BME and boiling. Reactions were visualized on a BioRad 4-20\% pre-cast SDS-PAGE stain-free gel using the BioRad ChemiDoc stain-free protocol or fluorescently using the Typhoon 9500 by Thermo. For 384-well plate analysis, proteins were treated as above, then eluted with $40 \mu \mathrm{L} 20 \mathrm{mM}$ Tris $\mathrm{pH} 7.5$, $150 \mathrm{mM} \mathrm{NaCl}, 2 \mathrm{mM}$ TCEP-HCl, and $1 \mathrm{M}$ Imidazole. $15 \mu \mathrm{L}$ of each fraction were loaded onto a 384-well clear-bottom microtiter plate and visualized on the Typhoon 9500. Samples from these fractions were also analyzed for metal content by ICP-MS.

Sod1, Ccs1, and Cu(I)-Ctr1c-Ccs1 affinity determination

H46R/H48Q/C146S Sod1 was fluorescently labeled with Alexa-546-C-maleimide and the reaction was quenched with DTT. Excess unbound dye was removed by gel filtration using a GE Sepharose S200 increase gel filtration column. Final Sod1-probe concentration in all assays was $10 \mathrm{nM}$. Ccs1 was titrated into reactions in increasing concentrations. We followed the HI-FI method to determine dissociation constants $\left(\mathrm{K}_{\mathrm{D}}\right)$ as outlined in previous publications (Winkler et al. 2012). Fluorescence quenching was visualized and $\mathrm{K}_{\mathrm{D}}$ and binding curves were determined with the GraphPad Prism software suite.

\section{Results}

Ctr1c stably binds Ces through

a $\mathrm{Cu}(\mathrm{I})$ intermediate

To focus upon the intracellular copper transfer between Ctr1 and Ccs, we utilized the cytosolic copper delivery domain of Ctr1 (Ctr1c) (Kahra et al. 2016). Ctr1c is a short 13 amino acid peptide that is difficult to visualize/quantitate biochemically, so a fluorescent label (Alexa 488) was conjugated to the $\mathrm{N}$-terminus. Labeled peptide $\left(\mathrm{Ctr} 1 \mathrm{c}_{488}\right)$ was purified and then visualized on Native-PAGE to confirm that non-native oligomerization did not occur (Fig. S1). Fluoro-labeling did not affect copper binding, as both labeled and unlabeled $\mathrm{Ctr} 1 \mathrm{c}$ peptides bound $\mathrm{Cu}(\mathrm{I})$ equivalently as determined by ICP-MS analysis (Table 1). The percentage of $\mathrm{Cu}(\mathrm{I})$ bound to $\mathrm{Ctr} 1 \mathrm{c}$ indicates that multiple $\mathrm{Ctr} 1 \mathrm{c}$ peptides bind a single copper at their $\mathrm{HCH}$ motifs, likely in a $3: 1-4: 1$ stoichiometry (Fig. 1a). We suggest that the 3:1 ratio is more likely as $\mathrm{Cu}(\mathrm{I})$ prefers to bind 2 or 3 ligands. Trimeric binding of $\mathrm{Ctr} 1 \mathrm{c}$ to copper also occurs in fulllength Ctr1. To determine if Ctr1c and Ccs would stably interact, both apo and $\mathrm{Cu}(\mathrm{I})$-loaded $\mathrm{Ctr} 1 \mathrm{c}_{488}$ were incubated with $\mathrm{Ccs}$ and visualized fluorescently on Native-PAGE. Apo-Ctr1 $\mathrm{c}_{488}$ did not bind to Ccs, but $\mathrm{Cu}(\mathrm{I})-\mathrm{Ctr} 1 \mathrm{c}_{488}$ formed a stable complex that ran as

Table 1 ICP-MS results shown as the percentage of copperbound protein

\begin{tabular}{lll}
\hline ICP-MS results & Protein:Cu(I) ratio \\
\hline Ctr1c & $22.6 \% \pm 1.4$ & $3-4: 1$ \\
Ctr1c 488 & $23.9 \% \pm 1.7$ & $3-4: 1$ \\
WT Ccs & $87.4 \% \pm 2.6$ & $1: 1$ \\
MXAXXA & $85.2 \% \pm 0.4$ & $1: 1$ \\
AXA & $82.0 \% \pm 1.2$ & $1: 1$ \\
\hline
\end{tabular}

The first two rows show the copper loading efficiency of the Alexa 488 labeled and non-labeled Ctr1c constructs. Percent bound remained consistent regardless of the amount of excess copper added. Final three rows describe the amount of copper bound to $\mathrm{Ccs}$ constructs after $\mathrm{Ni}$ pulldown assays with $\mathrm{Cu}(\mathrm{I})$ loaded $\mathrm{Ctr} 1 \mathrm{c}_{488}$ 


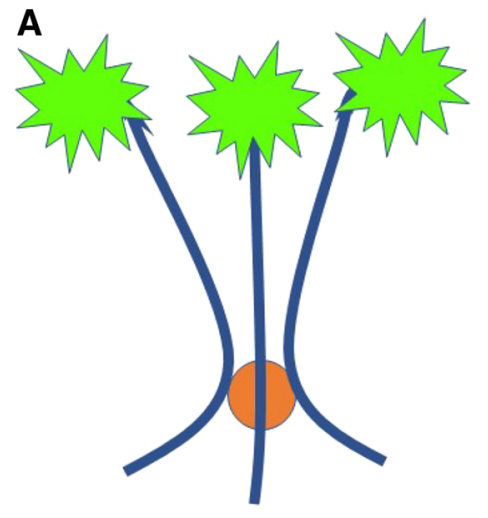

Fig. 1 Ctr1c binding to Ccs is copper dependent. a Copper (orange circle) is coordinated by three $\mathrm{Ctr} 1 \mathrm{c}_{488}$ peptides at their $\mathrm{C}$-terminal HCH motifs. The Alexa 488 dye is conjugated to the $\mathrm{N}$-terminus using a succinimidyl ester linkage. ICP-MS determined that $\mathrm{Ctr} 1 \mathrm{c}_{488}$ bound $\mathrm{Cu}(\mathrm{I})$ in a $3: 1$ stoichiometry,

a clear band on the gel (Fig. 1b, Lanes 1 and 2). However, Ctr1c could not form a similar interaction with Ccs' target (metal-free, disulfide reduced Sod1 $\left(\mathrm{E}, \mathrm{E}-\mathrm{Sod} 1^{\mathrm{SH}}\right)$ ). Incubation of $\mathrm{Cu}(\mathrm{I})-\mathrm{Ctr} 1 \mathrm{c}_{488}$ with E,ESod1 ${ }^{\mathrm{SH}}$ did not result in stable complex formation (Fig. 1b, Lane 3). Furthermore, the Sod1 from this reaction was subsequently analyzed for metal content by ICP-MS, and no direct copper transfer occurred between Ctr1c and Sod1.

Stable Ctr1c.Ccs interaction can form at either copper binding motif of Ccs

We use a high-throughput experimental setup that takes advantage of clear-bottom microplates for fluorescent-based visualization and quantification of pull-down results (detailed in the "Materials and Methods" section). Figure 2a shows an example of the pull-down assays performed to examine the role of the copper binding motifs in Ces in coordinating Ctr1c. His-tagged wild-type $\mathrm{Ccs}$ or $\mathrm{Ccs}$ variants with $\mathrm{Cu}(\mathrm{I})$ Ctr $1 \mathrm{c}_{488}$ (top row) were assayed and then normalized against a copper-null variant of $\mathrm{Ccs}$ that was also incubated with $\mathrm{Cu}(\mathrm{I})-\mathrm{Ctr} 1 \mathrm{c}_{488}$ (bottom row). Three experimental replicates were performed and quantified to determine relative binding propensity (Fig. 2b). There does not appear to be a strong preference for either copper binding site in Ccs (D1-MxCxxC or D3$\mathrm{CxC}$ ). The copper-mediated interaction between $\mathrm{Ctr} 1 \mathrm{c}$ and Ccs occurs as long as copper is bound by at least

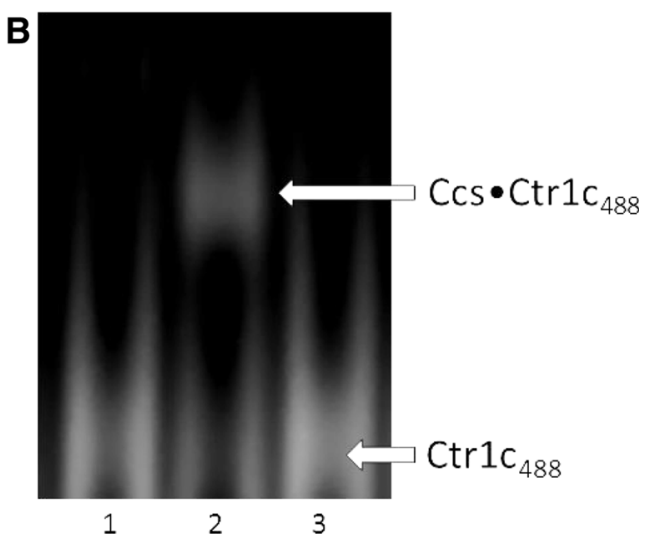

similar to full-length Ctr1. b Native PAGE gel imaged fluorescently for Alexa 488. Lane 1: $\mathrm{Ctr} 1 \mathrm{c}_{488}+\mathrm{Ccs}$. Lane 2: $\mathrm{Cu}(\mathrm{I})-\mathrm{Ctr}_{1} \mathrm{c}_{488}+\mathrm{Ccs}$. Lane 3: $\mathrm{Cu}(\mathrm{I})-\mathrm{Ctr}_{1 \mathrm{c}} \mathrm{C}_{88}+\mathrm{E}, \mathrm{E}-$ Sod $1^{\mathrm{SH}}$. (Color figure online)

one of these sites. Similar $\mathrm{Cu}(\mathrm{I})$ content was observed in all three samples by ICP-MS (Table 1). Incubating with $15 \mu \mathrm{M} \mathrm{Cu}(\mathrm{I})-\mathrm{Ctr} 1 \mathrm{c}_{488}$ consistently resulted in the recovery of $>4 \mu \mathrm{M}$ copper-bound Ccs, supporting the binding of copper by peptide in a $3: 1$ ratio. If the peptide to copper ratio was $4: 1$ the maximum amount of copper transferred to Ccs would be $3.75 \mu \mathrm{M}$. In vitro Sod1 activation assays performed using the $\mathrm{Cu}(\mathrm{I})-\mathrm{Ctr} 1 \mathrm{c}_{488} \cdot \mathrm{Ccs}$ containing samples showed that the complexes are functional and although $\mathrm{Cu}(\mathrm{I})$ Ctr $1 \mathrm{c}_{488}$ will coordinate with both copper-binding motifs in Ccs, only the CxC motif in Ccs D3 is critical for full activation of immature Sod1 under these conditions (Fig. 2c).

Copper-loaded Ctr1c associates stably with a Ccs.Sod 1 complex

To characterize possible Ctr1c. Ccs interactions with Sod1, similar pull-down assays utilizing a His-tagged $\mathrm{Ccs}$ in complex with $\mathrm{Cu}(\mathrm{I})-\mathrm{Ctr} 1 \mathrm{c}_{488}$ were conducted (Fig. 3). Immediate copper transfer and dissociation does not occur and a stable $\mathrm{Cu}(\mathrm{I})-\mathrm{Ctr} 1 \mathrm{c}_{488}$. Ccs complex is formed, as previously demonstrated (Fig. 1). The Sod1 molecule used in this assay is an engineered immature form of Sod1 that cannot bind copper at the active site or form its intra-subunit disulfide bond $\left(\mathrm{X}, \mathrm{Zn}\right.$-Sod $\left.1^{\mathrm{X}}\right)$, which is commonly used to form "stalled" Ccs.Sod1 complexes (Lamb et al. 2000, 2001; Winkler et al. 2009). Direct interaction 
A

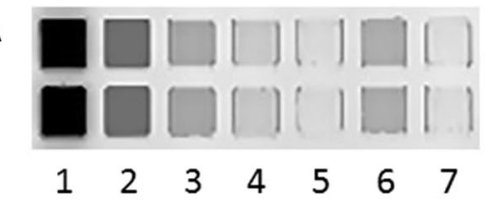

C

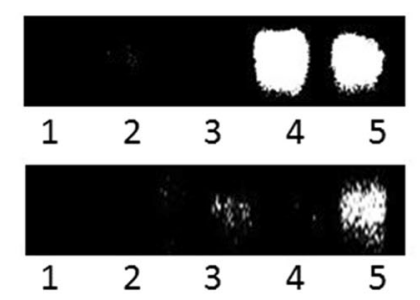

Fig. 2 Ctr1c can bind both copper binding domains of Ccs. a Nickel pulldown assays were loaded into a clear-bottom 384-well plate and visualized fluorescently using the Typhoon 9500. Lane 1: Input, Lane 2: Flow-through, Lanes 3-5: Washes, Lane 6: Elution, Lane 7: Buffer blank. b This graph of fluorescence intensity is normalized to mutant Ccs lacking copper binding motifs. (c, Top) Lane 1: Sod1 stripped of metals,

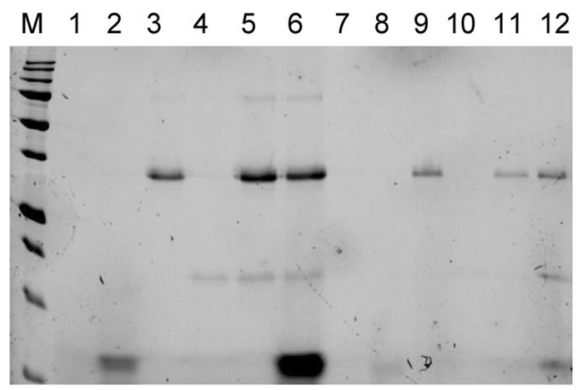

Fig. 3 Immature Sod1 can form a stable Sod1.Ccs.Ctr1c heterotrimeric complex. Lane 1: beads supernatant, Lane 2: $\mathrm{Cu}(\mathrm{I})-\mathrm{Ctr} 1 \mathrm{c}_{488}$ supernatant, Lane 3: His ${ }_{8}-\mathrm{Ccs}$ supernatant, Lane 4: Sod1 supernatant, Lane 5: $\mathrm{His}_{8}-\mathrm{Ccs} \cdot \mathrm{Sod} 1$ supernatant, Lane 6: $\mathrm{His}_{8}$-Ccs $\cdot \mathrm{Sod} 1$ and $\mathrm{Cu}(\mathrm{I})-\mathrm{Ctr} 1 \mathrm{c}_{488}$ supernatant, Lane 7: beads

between $\mathrm{Cu}(\mathrm{I})-\mathrm{Ctr} 1 \mathrm{c}_{488}$ and $\mathrm{X}, \mathrm{Zn}-\mathrm{Sod} 1^{\mathrm{X}}$ is not detected under any conditions tested (Figs. 1b, 2c). In addition, we show that a stable $\mathrm{Cu}(\mathrm{I})-\mathrm{Ctr} 1 \mathrm{c}_{488}$. Ccs complex can recognize and bind to $\mathrm{X}, \mathrm{Zn}-\mathrm{Sod} 1^{\mathrm{X}}$ in a similar manner to that of Ccs alone (Fig. 4b). Strikingly, $\mathrm{Cu}(\mathrm{I})-\mathrm{Ctr} 1 \mathrm{c}_{488}$ also interacts stably with a preformed $\mathrm{Ccs} \cdot \mathrm{X}, \mathrm{Zn}-\mathrm{Sod} 1^{\mathrm{X}}$ heterodimeric complex (Fig. 3, lane 12). The Ctr1c molecule does not dissociate upon Ccs binding to Sod1 and forms a $\mathrm{Cu}(\mathrm{I})-\mathrm{Ctr} 1 \mathrm{c}_{488} \cdot \mathrm{Ccs} \cdot \mathrm{X}, \mathrm{Zn}-\mathrm{Sod} 1^{\mathrm{X}}$ heterotrimeric complex, supporting multiple delivery options arising from the Ctr1 transporter.

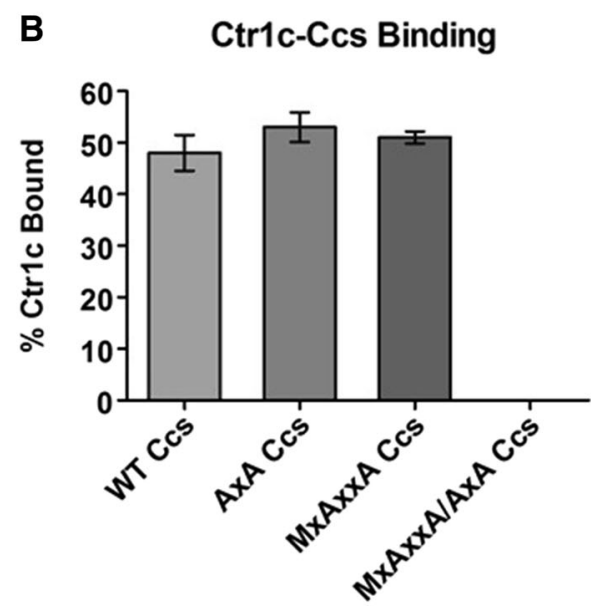

Lane 2: Sod1 with $\mathrm{Cu}(\mathrm{I})$ - Ctr1c, Lane 3: Sod1 with apoMxAxxA Ccs, Lane 4: Sod1 with apo-MxAxxA Ccs and Cu(I)Ctr1c, Lane 5: Sod1 with Cu(1)- WT Ccs. (C, Bottom) Lane 1: Sod1 stripped of metals, Lane 2: Sod1 with Cu(I)-Ctr1c, Lane 3: Sod1 with apo-AxA Ccs, Lane 4: Sod1 with apo-AxA Ccs and $\mathrm{Cu}(\mathrm{I})-\mathrm{Ctr} 1 \mathrm{c}$, Lane 5: Sod1 with $\mathrm{Cu}(1)-\mathrm{WT}$ Ccs

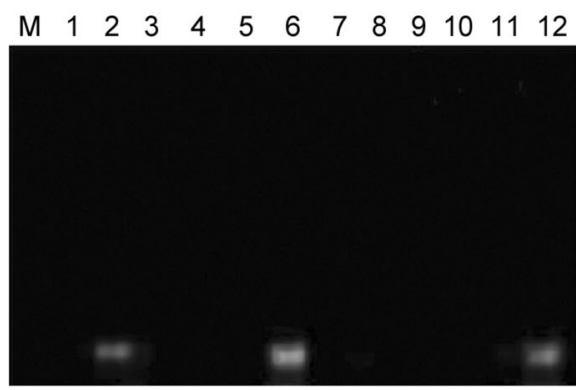

elution, Lane 8: $\mathrm{Cu}(\mathrm{I})-\mathrm{Ctr} 1 \mathrm{c}_{488}$ elution, Lane 9: His ${ }_{8}-\mathrm{Ccs}$ elution, Lane 10: Sod1 elution, Lane 11: $\mathrm{His}_{8}-\mathrm{Ccs} \cdot \mathrm{Sod} 1$ elution, Lane 12: $\mathrm{His}_{8}-\mathrm{Ccs} \cdot \mathrm{Sod} 1$ and $\mathrm{Cu}(\mathrm{I})-\mathrm{Ctr} 1 \mathrm{c}_{488}$ elution. The right image is the same gel as the left (stain free), but visualized fluorescently

Ces association with Ctr1c does NOT affect its affinity for Sod1

The assumed order of operation for Sod1 activation starts with the metalation of Ccs, which then finds/ binds an immature Sod1 molecule to activate (see reviews Culotta, Lin et al. 1999; Rosenzweig 2001; Furukawa and O'Halloran 2006; Leitch et al. 2009; Robinson and Winge 2010; Fetherolf et al. 2017a, b). Much like the yeast Ccs.Sod1 interaction (Boyd et al. 2018), human Ccs favors binding to completely immature Sod1 (E,E-Sod1 ${ }^{\mathrm{SH}}$ ) (Fig. 4a). The copper 


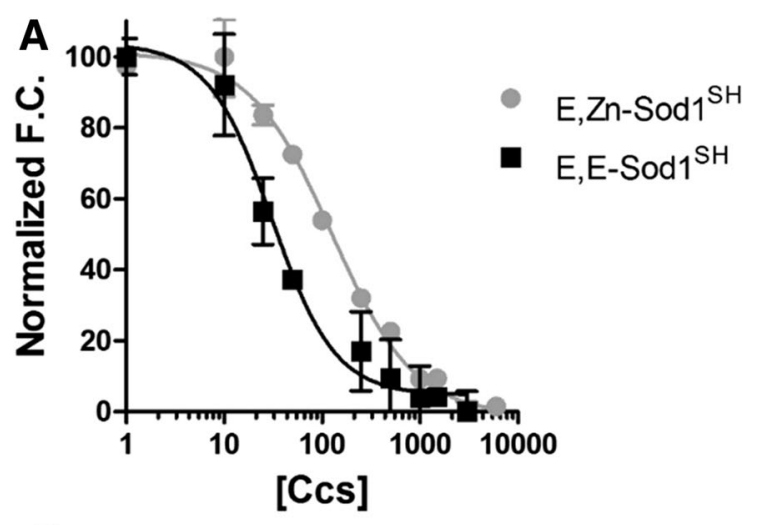

$\mathrm{Cu}(\mathrm{I})-\mathrm{Ctr} 1 \mathrm{c}$ cannot directly activate $\mathrm{Sod} 1$, alone, but requires the $\mathrm{Cu}(\mathrm{I})-\mathrm{Ctr1c} \cdot \mathrm{Ccs}$ complex (Fig. 4c, Lanes 4 and 2, respectively). Control gels of the reactions were visualized by multiple approaches to confirm equal loading (Fig. S2). Our results continue to support the idea that an entire copper-trafficking pathway can, but is not necessitated to occur within a single complex, yet the question arises as to what finally triggers eventual dissociation.

Complete Sod1 activation terminates

the Sod1.Ccs.Ctr1c association

B

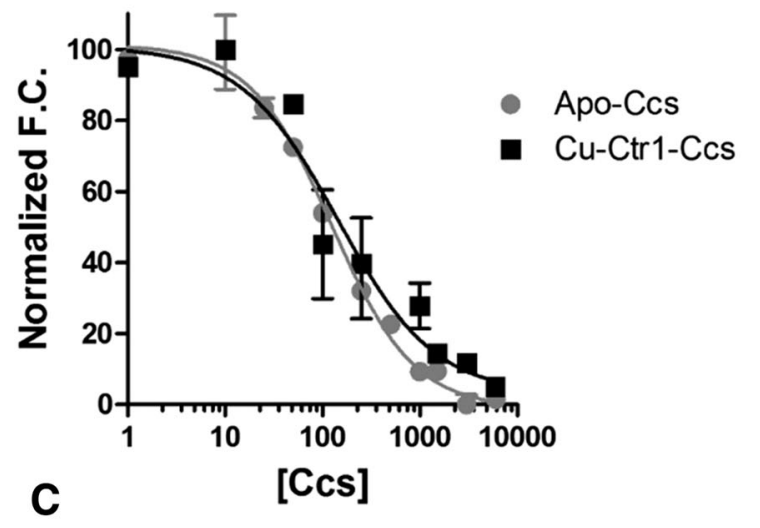

To study dissociation of the $\mathrm{Cu}(\mathrm{I})-\mathrm{Ctr} 1 \mathrm{c} \cdot \mathrm{Ccs} \cdot \mathrm{Sod} 1$ complex, we performed pulldowns now using immature Sod1 (E,Zn-Sod1 ${ }^{\mathrm{SH}}$ ) (Fig. 5a). When E,ZnSod $1{ }^{\mathrm{SH}}$ was added to a pre-formed $\mathrm{Cu}(\mathrm{I})-\mathrm{Ctr} 1 \mathrm{c}_{488} \cdot \mathrm{Ccs}$ complex, immediate and complete dissociation of the complex resulted (Fig. 5a, Lane 14). Activation assays were performed on samples obtained from the pulldowns and the E,Zn-Sod1 ${ }^{\mathrm{SH}}$ that had been allowed to interact with $\mathrm{Cu}(\mathrm{I})-\mathrm{Ctr} 1 \mathrm{c} \cdot \mathrm{Ccs}$ complex was analyzed and had activity comparable to that of E,Zn-Sod $1^{\mathrm{SH}}$ incubated with $\mathrm{Cu}(\mathrm{I})-\mathrm{Ccs}$, alone (i.e. the Sod1 is now fully mature $\left(\mathrm{Cu}, \mathrm{Zn}\right.$-Sod $\left.\left.1^{\mathrm{SS}}\right)\right)$. E,Zn-Sod $1^{\mathrm{SH}}$ incubated with $\mathrm{Cu}(\mathrm{I})-\mathrm{Ctr} 1 \mathrm{c}$ alone did not have activity, nor did the immaturely-trapped $\mathrm{X}, \mathrm{Zn}$-Sod $1^{\mathrm{X}}$ mutant that was complexed with $\mathrm{Cu}(\mathrm{I})-\mathrm{Ctr} 1 \mathrm{c} \cdot \mathrm{Ccs}$ (Fig. 5b). This clearly indicates that the $\mathrm{Cu}(\mathrm{I})$-Ccs.Ctr1c complex can activate immature $\mathrm{Sod} 1$ in a way that $\mathrm{Cu}(\mathrm{I})$ Ctr1c cannot accomplish (Fig. 4c, Lanes 4 and 2, respectively). Only complete Sod 1 activation triggers dissociation of the stable $\mathrm{Cu}(\mathrm{I})-\mathrm{Ctr} 1 \mathrm{c} \cdot \mathrm{Ccs} \cdot \mathrm{Sod} 1 \mathrm{com}-$ plex, suggesting that the portion of Sod1 activated through this direct Ctr1-to-Ccs-to-Sod1 pathway occurs while tethered to the cell membrane via the complete $\mathrm{Ctr} 1 \mathrm{Cu}$-transporter.

\section{Discussion}

occupancy of Ccs has a negligible role in Sod1 binding affinity, as the copper-loaded form of Ces binds to E,E-Sod $1^{\mathrm{SH}}$ in a nearly indistinguishable fashion. Additionally, a pre-made $\mathrm{Cu}(\mathrm{I})-\mathrm{Ctr} 1 \mathrm{c} \cdot \mathrm{Ccs}$ complex binds E,E-Sod $1^{\mathrm{SH}}$ with a similar affinity to both apo and copper-bound Ccs (Fig. 4b). Again, we also wanted to ensure that the $\mathrm{Cu}(\mathrm{I})-\mathrm{Ctr} 1 \mathrm{c} \cdot \mathrm{Ccs}$ complex assembled is functional and can fully activate immature Sod1. In vitro Sod1 activation assays reveal that

Up until now, a considerable amount of work has been completed on the Ccs-mediated Sod1 activation process (reviewed in Seetharaman et al. 2009), yet details of copper transfer to Ccs were still unclear. Our data shows that the intracellular C-terminal tail of the influx transporter $\mathrm{Ctr} 1$ (Ctr1c) forms a stable coppermediated interaction with Ccs, as complete copper transfer does not immediately follow first contact. The 

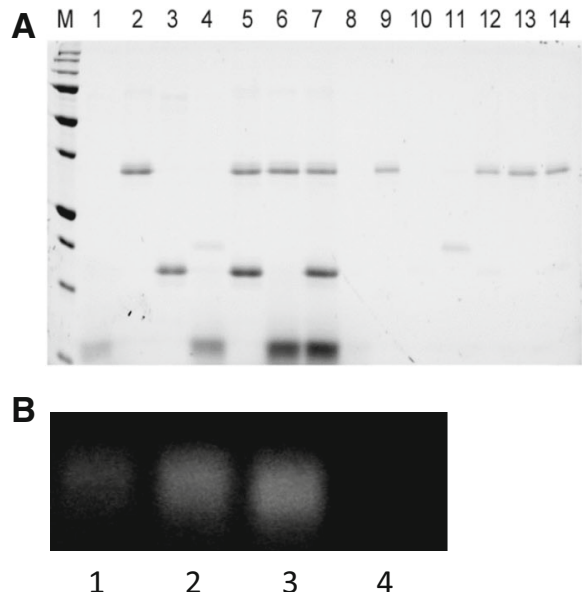

Fig. 5 Sod1 maturation disassociates the Sod1.Ccs. Cu(I)Ctr1c complex. Lane 1: $\mathrm{Cu}(\mathrm{I})-\mathrm{Ctr} 1 \mathrm{c}_{488}$ supernatant, Lane 2: $\mathrm{His}_{8}-\mathrm{Ccs}$ supernatant, Lane 3: Sod1 supernatant, Lane 4: $\mathrm{His}_{8}-$ Sod1 with $\mathrm{Cu}(\mathrm{I})-\mathrm{Ctr} 1 \mathrm{c}_{488}$ supernatant, Lane 5: His 8 -Ccs-Sod1 supernatant, Lane 6: $\mathrm{His}_{8}-\mathrm{Ccs}$ with $\mathrm{Cu}(\mathrm{I})-\mathrm{Ctr} 1 \mathrm{c}_{488}$ supernatant, Lane 7: $\mathrm{His}_{8}-\mathrm{Ccs} \cdot \mathrm{Sod} 1$ with $\mathrm{Cu}(\mathrm{I})-\mathrm{Ctr} 1 \mathrm{c}_{488}$ supernatant, Lane 8: $\mathrm{Cu}(\mathrm{I})-\mathrm{Ctr} 1 \mathrm{c}_{488}$ elution, Lane 9: His ${ }_{8}-\mathrm{Ccs}$ elution, Lane 10: Sod1 elution, Lane 11: $\mathrm{His}_{8}-\mathrm{Sod} 1$ with $\mathrm{Cu}(\mathrm{I})-\mathrm{Ctr} 1 \mathrm{c}_{488}$ elution, Lane 12: His 8 -Ccs.Sod1 elution, Lane 13: $\mathrm{His}_{8}-\mathrm{Ccs}$ with $\mathrm{Cu}(\mathrm{I})-$

introduction of an engineered Sod1 variant that cannot bind copper or form its conserved intra-subunit disulfide bond (X, Zn-Sod1 ${ }^{\mathrm{X}}$ ) generates a heterotrimeric $\mathrm{Cu}(\mathrm{I})-\mathrm{Ctr} 1 \mathrm{c} \cdot \mathrm{Ccs} \cdot \mathrm{Sod} 1$ complex. Only complete Sod1 activation severs the ties between transporter, chaperone, and target. Together, the results presented above provide clear evidence for a complete copper delivery pathway held within a singular 3-part complex.

The Unger laboratory was the first to show that yeast Ccs1 interacts directly with Ctr1 (Pope et al. 2013). Their work highlighted that yCcs1 can engage with lipid bilayers and suggested that recruitment to the plasma membrane is an essential step in Ccsmediated Sod1 activation (Pope et al. 2013). The authors of that study submit mechanistic ambiguities in the proposed process and how further investigation would be needed to "unravel" these details. In the present study, we have taken a much narrower focus upon the intracellular C-terminal domain of Ctr1 (Ctr1c). This domain is known to be important for the coordination and eventual distribution of copper to trafficking molecules within the cytosol (Xiao and Wedd 2002; Kahra et al. 2016). We show that this domain alone is enough to interact with Ccs or a Ccs.Sod 1 complex, and the interactions are entirely

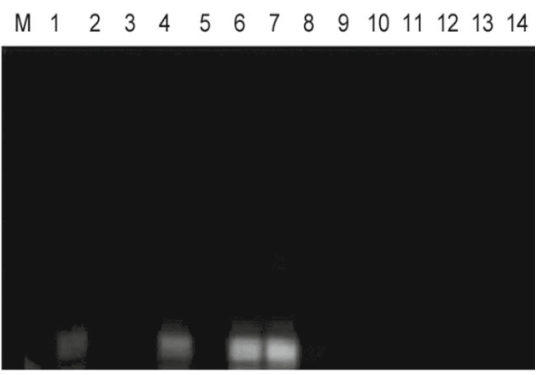

Ctr1 $\mathrm{c}_{488}$ elution, Lane 14: $\mathrm{His}_{8}-\mathrm{Ccs} \cdot \mathrm{Sod} 1$ with $\mathrm{Cu}(\mathrm{I})-\mathrm{Ctr} 1 \mathrm{c}_{488}$ elution. The right image is the same gel as the left (stain free), but visualized fluorescently. b Lane 1: $\mathrm{Cu}(\mathrm{I})$-loaded $\mathrm{Ctr}_{1 \mathrm{c}_{488}}$ + WT Sod1 from a, Lane 4. Lane 2: Cu(I)-loaded WT His $8^{-}$ Ccs + WT Sod1 set up separately as a positive control. Lane 3: WT His 8 -Ccs + WT Sod1 + Cu(I)-Ctr1 $\mathrm{c}_{488}$ from a, Lane 7. Lane 4: WT $\mathrm{His}_{8}-\mathrm{Ccs}+$ immature Sod1 + Cu(I)-Ctr1 $\mathrm{c}_{488}$ from Fig. 3, Lane 12

copper dependent (Figs. 1, 4), much like for the complete Ctr1 transporter (Pope et al. 2013). Similar results have been demonstrated for $\mathrm{Ctr} 1 \mathrm{c}$ and the copper chaperone Atx1 (Xiao and Wedd 2002), which shares similarity with D1 of Ccs.

Numerous groups, including our own, have previously shown that Ccs recognizes and binds immature forms of Sod1 (Banci et al. 2012; Wright et al. 2016; Fetherolf et al. 2017a, b; Luchinat et al. 2017; Boyd et al. 2018). The Sod1-like D2 of Ccs directs this interaction with D3 contributing to high-affinity binding and full activation (Boyd et al. 2018). More recently, we have demonstrated that copper binding by Ces does not significantly affect the Sod1 interaction (Boyd et al. 2018). The question then arose as to whether the $\mathrm{Cu}(\mathrm{I})-\mathrm{Ctr} 1 \mathrm{c} \cdot \mathrm{Ccs}$ interaction alters $\mathrm{Ccs}$ recognition and binding to Sod1. Upon examination, Ccs can recognize and bind immature Sod 1 while still coordinating copper with Ctr1c. In fact, a stable highaffinity $\mathrm{Cu}(\mathrm{I})-\mathrm{Ctr} 1 \mathrm{c} \cdot \mathrm{Ccs} \cdot \mathrm{Sod} 1$ complex is observed (Figs. 2, 3,4). The next logical steps were to test if this heterotrimeric complex results in activation of Sod1.

Ccs-mediated Sod1 activation is an intricate process involving fold-induced zinc binding by Sod 1 that is followed by disulfide bond driven copper delivery (Culotta et al. 1997, 2006). Multiple lines of evidence 


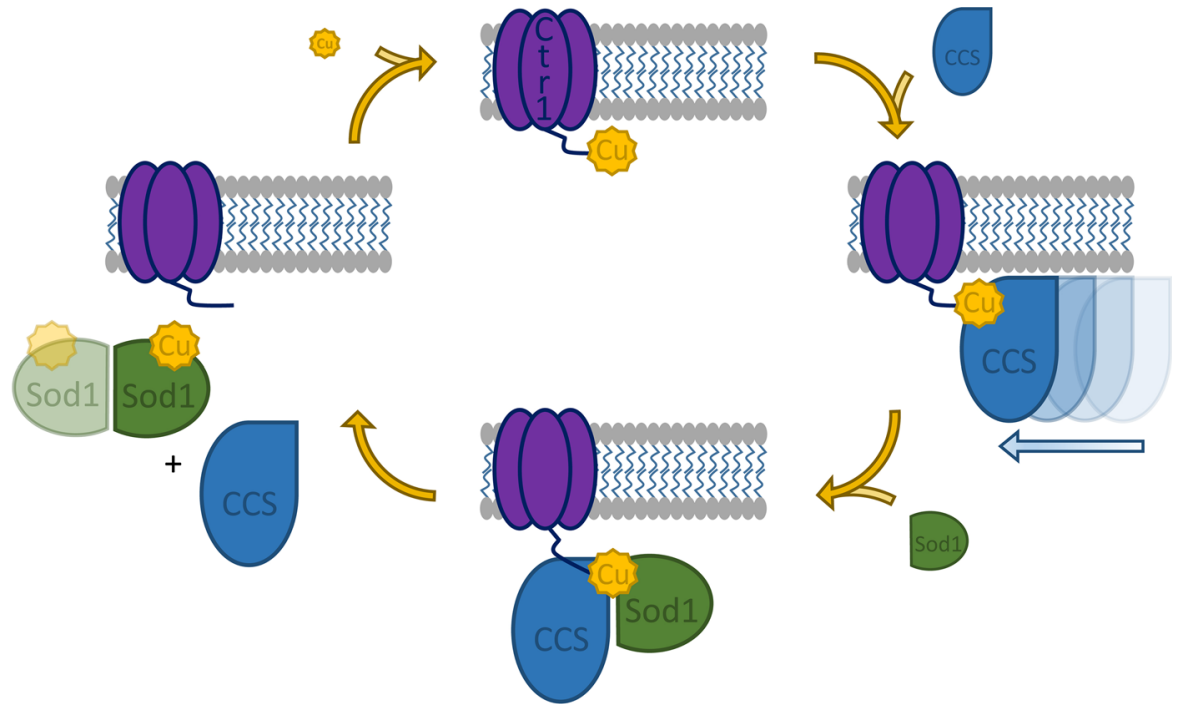

Fig. 6 Model of Ccs copper acquisition from Ctr1 and complex disassembly by Sod1 maturation. Ctr1 (purple) transports copper (yellow) into the cytosol. Ccs (blue) scans the membrane and associates with the copper-bound $\mathrm{Ctr} 1 \mathrm{C}$-terminal domain.

suggest that cellular conditions may necessitate the role(s) for Ccs and that ancillary molecules like reduced glutathione (GSH) may facilitate the activation process (Maryon et al. 2013). In fact, GSH has been shown to promote an entirely Ccs-independent Sod1 activation pathway in mammals (Leitch et al. 2009). The $\mathrm{Cu}(\mathrm{I})-\mathrm{Ctr} 1 \mathrm{c}$ construct will not donate $\mathrm{Cu}(\mathrm{I})$ to $\mathrm{Sod} 1$ directly, but a preformed $\mathrm{Cu}(\mathrm{I})$ Ctr1c.Ccs complex can fully activate immature Sod1 in vitro (Fig. 5b). This excludes a Ccs-independent mechanism of Sod1 activation catalyzed directly by Ctr1c.

It is quite intriguing that the $\mathrm{Cu}(\mathrm{I})-\mathrm{Ctr} 1 \mathrm{c} \cdot \mathrm{Ccs} \cdot \mathrm{Sod} 1$ complex is not transiently associated. This suggests that a simple $\mathrm{Cu}(\mathrm{I})$ hand-off between $\mathrm{Ctr} 1 \mathrm{c}$ and $\mathrm{Ccs}$ is not occurring; so, what induces eventual dissociation? The Ccs·Sod1 interaction, without Ctr1c, has also been shown to be remarkably stable as long as copper cannot be delivered to the Sod1 active site (Fetherolf et al. 2017a, b). This can be ensured in two ways: (1) copper is not provided to the complex or (2) the active site is modified so that the bound copper cannot be delivered to that site (Fetherolf et al. 2017a, b). We have previously shown that Ccs delivers copper to an "entry-site" near the Sod1.Ccs interface (Fetherolf et al. 2017a, b). Oxygen dependent disulfide bond formation eliminates this site, promotes copper
Sod1 (green) interacts with the $\mathrm{Cu}(\mathrm{I})-\mathrm{Ctr} 1-\mathrm{Ccs}$ complex. Sod1 maturation terminates the ternary complex. Ccs is allowed to cycle through the copper-acquisition process again. The Sod1 homo-dimer is fully active. (Color figure online)

shuttling to the nearby active site, and terminates interaction with Ccs (Fetherolf et al. 2017a, b). Copper must be present to form the $\mathrm{Cu}(\mathrm{I})-\mathrm{Ctr} 1 \mathrm{c} \cdot \mathrm{Ccs} \cdot \mathrm{Sod} 1$ heterotrimer, but the Sod1 active site must be ablated or the complex readily activates Sod1 and the complex promptly dissolves. This likely indicates that $\mathrm{Ctr} 1 \mathrm{c}$ is co-coordinating the copper ion up until disulfide bond formation in Sod1 disperses the complex producing $\mathrm{Cu}, \mathrm{Zn}-\mathrm{Sod} 1^{\mathrm{SS}}$ that can now homodimerize along with copper-free forms of both $\mathrm{Ccs}$ and $\mathrm{Ctr} 1 \mathrm{c}$ that no longer have any affinity for each other (Fig. 6).

Noteworthy is that Pope et al. showed that yeast Ccs1 strongly associates with lipid bilayers, but that the yCcs1·ySod 1 heterodimer reduced this propensity most likely due to a critical positively charged patch on yCcs1 that is not present on ySod1 (Pope et al. 2013). This observation may explain how Ces first comes into contact with $\mathrm{Cu}(\mathrm{I})-\mathrm{Ctr} 1$, and how the products of this reaction (e.g. activated Sod1 and apoCcs) are provided release from the membrane to freely access other cellular locations. Together, this present study establishes an all-inclusive design for Ctr1-toCcs-to-Sod1 copper delivery and provides new evidence for a singular heterotrimeric complex forming the foundation for the pathway.

Acknowledgements This research was supported in part by R01 GM120252 from the National Institutes of Health awarded 
to D.D.W. The content is solely the responsibility of the authors and does not necessarily represent the official views of the National Institutes of Health.

\section{Compliance with ethical standards}

Conflicts of interest The authors declare that they have no conflicts of interest with the contents of this article.

Open Access This article is distributed under the terms of the Creative Commons Attribution 4.0 International License (http:// creativecommons.org/licenses/by/4.0/), which permits unrestricted use, distribution, and reproduction in any medium, provided you give appropriate credit to the original author(s) and the source, provide a link to the Creative Commons license, and indicate if changes were made.

\section{References}

Banci L, Bertini I, Cantini F, Kozyreva T, Massagni C, Palumaa P, Rubino JT, Zovo K (2012) Human superoxide dismutase 1 (hSOD1) maturation through interaction with human copper chaperone for SOD1 (hCCS). Proc Natl Acad Sci USA 109:13555-13560

Banci L, Cantini F et al (2013) Mechanistic aspects of hSOD1 maturation from the solution structure of $\mathrm{Cu}(\mathrm{I})$-loaded hCCS domain 1 and analysis of disulfide-free hSOD1 mutants. ChemBioChem 14(14):1839-1844

Boyd SD, Liu L et al (2018) Quantifying the interaction between copper-zinc superoxide dismutase (Sod1) and its copper chaperone (Ccs1). J Proteom Bioinform 11(4):473

Bremner I (1998) Manifestations of copper excess. Am J Clin Nutr 67(5 Suppl):1069S-1073S

Culotta VC, Klomp LW et al (1997) The copper chaperone for superoxide dismutase. J Biol Chem 272(38):23469-23472

Culotta VC, Lin SJ et al (1999) Intracellular pathways of copper trafficking in yeast and humans. Adv Exp Med Biol 448:247-254

Culotta VC, Yang M et al (2006) Activation of superoxide dismutases: putting the metal to the pedal. Biochim Biophys Acta 1763(7):747-758

Dancis A, Haile D et al (1994) The Saccharomyces cerevisiae copper transport protein $(\mathrm{Ctr} 1 \mathrm{p})$. Biochemical characterization, regulation by copper, and physiologic role in copper uptake. J Biol Chem 269(41):25660-25667

De Feo CJ, Aller SG et al (2009) Three-dimensional structure of the human copper transporter hCTR1. Proc Natl Acad Sci USA 106(11):4237-4242

Eisses JF, Kaplan JH (2002) Molecular characterization of hCTR1, the human copper uptake protein. J Biol Chem 277(32):29162-29171

Fetherolf MM, Boyd SD et al (2017a) Copper-zinc superoxide dismutase is activated through a sulfenic acid intermediate at a copper ion entry site. J Biol Chem 292(29):12025-12040
Fetherolf MM, Boyd SD et al (2017b) Oxygen-dependent activation of $\mathrm{Cu}, \mathrm{Zn}$-superoxide dismutase-1. Metallomics 9(8):1047-1059

Flores AG, Unger VM (2013) Atox1 contains positive residues that mediate membrane association and aid subsequent copper loading. J Membr Biol 246(12):903-913

Furukawa Y, O'Halloran TV (2006) Posttranslational modifications in $\mathrm{Cu}, \mathrm{Zn}$-superoxide dismutase and mutations associated with amyotrophic lateral sclerosis. Antioxid Redox Signal 8(5-6):847-867

Halliwell B, Gutteridge JM (1990) Role of free radicals and catalytic metal ions in human disease: an overview. Methods Enzymol 186:1-85

Hussain F, Olson JS et al (2008) Conserved residues modulate copper release in human copper chaperone Atox1. Proc Natl Acad Sci USA 105(32):11158-11163

Hussain F, Rodriguez-Granillo A et al (2009) Lysine-60 in copper chaperone Atox 1 plays an essential role in adduct formation with a target Wilson disease domain. J Am Chem Soc 131(45):16371-16373

Kahra D, Kovermann M et al (2016) The C-terminus of human copper importer $\mathrm{Ctr} 1$ acts as a binding site and transfers copper to Atox1. Biophys J 110(1):95-102

Kaplan JH, Maryon EB (2016) How mammalian cells acquire copper: an essential but potentially toxic metal. Biophys J 110(1):7-13

Kim BE, Nevitt T et al (2008) Mechanisms for copper acquisition, distribution and regulation. Nat Chem Biol 4(3): 176-185

Klomp AE, Juijn JA et al (2003) The N-terminus of the human copper transporter 1 (hCTR1) is localized extracellularly, and interacts with itself. Biochem J 370(Pt 3):881-889

Lamb AL, Wernimont AK et al (1999) Crystal structure of the copper chaperone for superoxide dismutase. Nat Struct Biol 6(8):724-729

Lamb AL, Torres AS et al (2000a) Heterodimer formation between superoxide dismutase and its copper chaperone. Biochemistry 39(48):14720-14727

Lamb AL, Wernimont AK et al (2000b) Crystal structure of the second domain of the human copper chaperone for superoxide dismutase. Biochemistry 39(7):1589-1595

Lamb AL, Torres AS et al (2001) Heterodimeric structure of superoxide dismutase in complex with its metallochaperone. Nat Struct Biol 8(9):751-755

Lee J, Pena MM et al (2002) Biochemical characterization of the human copper transporter Ctr1. J Biol Chem 277(6):4380-4387

Leitch JM, Yick PJ et al (2009) The right to choose: multiple pathways for activating copper, zinc superoxide dismutase. J Biol Chem 284(37):24679-24683

Luchinat E, Barbieri L, Banci L (2017) A molecular chaperone activity of CCS restores the maturation of SOD1 fALS mutants. Sci Rep 7:17433

Lutsenko S, Efremov RG et al (2002) Human copper-transporting ATPase ATP7B (the Wilson's disease protein): biochemical properties and regulation. J Bioenerg Biomembr 34(5):351-362

Maryon EB, Molloy SA et al (2013) Cellular glutathione plays a key role in copper uptake mediated by human copper transporter 1. Am J Physiol Cell Physiol 304(8):C768C779 
O'Halloran TV, Culotta VC (2000) Metallochaperones, an intracellular shuttle service for metal ions. J Biol Chem 275(33):25057-25060

Ohrvik H, Thiele DJ (2014) How copper traverses cellular membranes through the mammalian copper transporter 1, Ctr1. Ann NY Acad Sci 1314:32-41

Pena MM, Lee J et al (1999) A delicate balance: homeostatic control of copper uptake and distribution. J Nutr 129(7):1251-1260

Pickering IJ, George GN, Dameron CT, Kurz B, Winge DR, Dance IG (1993) X-ray absorption spectroscopy of cuprous-thiolate clusters in proteins and model systems. J Am Chem Soc 115:9498-9505

Pope CR, De Feo CJ et al (2013) Cellular distribution of copper to superoxide dismutase involves scaffolding by membranes. Proc Natl Acad Sci USA 110(51):20491-20496

Puig S, Lee J et al (2002) Biochemical and genetic analyses of yeast and human high affinity copper transporters suggest a conserved mechanism for copper uptake. J Biol Chem 277(29):26021-26030

Robinson NJ, Winge DR (2010) Copper metallochaperones. Annu Rev Biochem 79:537-562

Rodriguez-Granillo A, Wittung-Stafshede P (2008) Structure and dynamics of $\mathrm{Cu}(\mathrm{I})$ binding in copper chaperones Atox 1 and CopZ: a computer simulation study. J Phys Chem B 112(15):4583-4593

Rodriguez-Granillo A, Wittung-Stafshede P (2009) Differential roles of Met10, Thr11, and Lys60 in structural dynamics of human copper chaperone Atox1. Biochemistry 48(5):960-972

Rodriguez-Granillo A, Crespo A et al (2010) Copper-transfer mechanism from the human chaperone Atox1 to a metalbinding domain of Wilson disease protein. J Phys Chem B 114(10):3698-3706

Rosenzweig AC (2001) Copper delivery by metallochaperone proteins. Acc Chem Res 34(2):119-128

Rosenzweig AC, O'Halloran TV (2000) Structure and chemistry of the copper chaperone proteins. Curr Opin Chem Biol 4(2):140-147
Sala FA, Antonyuk SV, Garratt RC, Hasnain SS (2019) Molecular recognition and maturation of SOD1 by its evolutionarily destabilised cognate chaperone hCCS. PLoS Biol 17(2):e3000141

Schmidt PJ, Rae TD et al (1999) Multiple protein domains contribute to the action of the copper chaperone for superoxide dismutase. J Biol Chem 274(34):23719-23725

Schmidt PJ, Kunst C et al (2000) Copper activation of superoxide dismutase 1 (SOD1) in vivo. Role for protein-protein interactions with the copper chaperone for SOD1. J Biol Chem 275(43):33771-33776

Seetharaman SV, Prudencio M et al (2009) Immature copperzinc superoxide dismutase and familial amyotrophic lateral sclerosis. Exp Biol Med (Maywood) 234(10):1140-1154

Voskoboinik I, Camakaris J (2002) Menkes copper-translocating P-type ATPase (ATP7A): biochemical and cell biology properties, and role in Menkes disease. J Bioenerg Biomembr 34(5):363-371

Winkler DD, Schuermann JP et al (2009) Structural and biophysical properties of the pathogenic SOD1 variant H46R/ H48Q. Biochemistry 48(15):3436-3447

Winkler DD, Luger K et al (2012) Quantifying chromatin-associated interactions: the HI-FI system. Methods Enzymol 512:243-274

Wong PC, Waggoner D et al (2000) Copper chaperone for superoxide dismutase is essential to activate mammalian $\mathrm{Cu} / \mathrm{Zn}$ superoxide dismutase. Proc Natl Acad Sci USA 97(6):2886-2891

Wright GS, Antonyuk SV, Hasnain SS (2016) A faulty interaction between SOD1 and hCCS in neurodegenerative disease. Sci Rep 6:27691

Xiao Z, Wedd AG (2002) A C-terminal domain of the membrane copper pump Ctr1 exchanges copper(I) with the copper chaperone Atx1. Chem Commun (Camb) $6: 588-589$

Publisher's Note Springer Nature remains neutral with regard to jurisdictional claims in published maps and institutional affiliations. 